\title{
INFLUENCE OF GEOMETRIC CHARACTERISTICS OF CANAL ON PRECIPITATING CAPACITY OF SEPARATOR
}

\author{
L. A. Belyaev ${ }^{1}, A$. S. Zaitsev ${ }^{1}, A$. A. Kondakov ${ }^{1, *}, S$. A. Shevelev ${ }^{1}$, and $A$. S. Matveev ${ }^{1}$ \\ ${ }^{1}$ National Research Tomsk Polytechnic University, 634050 Tomsk, Russia
}

\begin{abstract}
Designed mathematic model allows calculating trajectories of spherical non-deformable particles movement in the flow of viscous nonburnt liquid in the flat canal of complex geometry. According to the results of calculations graphs of dependence of precipitating particles share on their diameter and degree of curvature of the researched canal have been built.
\end{abstract}

\section{Introduction}

Economical efficiency and reliability of turbines work operating in the area below the curve of saturation mostly depends on moisture of vapor. Increase of final moisture leads to decrease of relative internal coefficient of efficiency and increased erosive wear of wheel space [1].

Different ways of vapor separation are used for reliable and effective work of turbine. One of the most popular ways is vapor separation in separators of louvered type.

The present work considers movement of two phase area consisting of non-burnt liquid and discrete phase in the form of separate spherical drops. Movement of the area occurs in the canal with smooth wave surface.

The purpose of the work is mathematic modeling of movement of drop moisture in the canal with set geometry and study of the process of precipitating at different canal curvature.

\section{Mathematical model and study technique}

Spherical non-deformable particle with known position, rate vector in the initial time and physical properties flies into the flat canal, principal scheme of which is depicted in picture 1. Then, the particle comes through the canal smoothly or under the influence of the area flow or actively interacts with solid surface and precipitates.

\footnotetext{
*Corresponding author: kondakov-alexandr@rambler.ru
} 


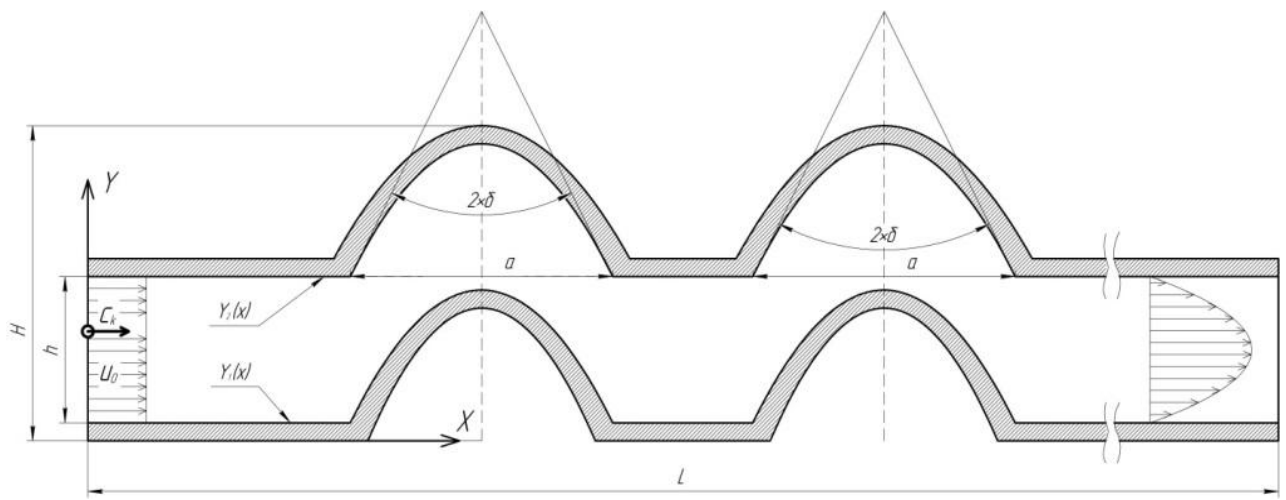

Fig. 1. Principal diagram of the researched canal.

Walls of the canal are set with functional dependences $\mathrm{Y}_{1}(\mathrm{x})$ and $\mathrm{Y}_{2}(\mathrm{x})$. Geometric characteristics of canal a, h, L are constant, and curvature of canal is determined by angle $\delta$.

Let us consider flat movement in Descartes system of coordinates, where only aerodynamic resistance force influences a particle [2].

Equation of particle movement in projections on the axis of coordinates for the case has the following view [2]:

$$
\begin{aligned}
& m \frac{d c_{k x}}{d t}=\rho F_{m . \text { e. }} c_{x}\left(u-c_{k x}\right) \sqrt{\left(u-c_{k x}\right)^{2}+\left(v-c_{k y}\right)^{2}} \\
& m \frac{d c_{k y}}{d t}=\rho F_{m . \text { e. }}{ }^{c}\left(v-c_{k y}\right) \sqrt{\left(u-c_{k x}\right)^{2}+\left(v-c_{k y}\right)^{2}}
\end{aligned}
$$

where

$c_{k x}, c_{k y}$ - vector projections of particle rate on the axis of coordinates;

$u, v$ - vector projection of flow rate on the axis of coordinates;

$m$ - drop mass;

$\rho$ - area density;

$c_{x}$ - coefficient of hydro mechanical resistance of particles which depends on Reynolds number;

$F_{m . \text { e. }}=\frac{\pi d_{e}^{2}}{4}$ - area of midship cross section.

Flow area of continuum is described with the help of the system of differential equations of Navier-Stocks for flat stationary laminar flow [3], which consists of two equations of movement quantity and equation of continuity. 


$$
\left\{\begin{array}{l}
\frac{\partial(\rho u u)}{\partial x}+\frac{\partial(\rho v u)}{\partial y}=\frac{\partial}{\partial x}\left(\mu \frac{\partial u}{\partial x}\right)+\frac{\partial}{\partial y}\left(\mu \frac{\partial u}{\partial y}\right)-\frac{\partial P}{\partial x} \\
\frac{\partial(\rho u v)}{\partial x}+\frac{\partial(\rho v v)}{\partial y}=\frac{\partial}{\partial x}\left(\mu \frac{\partial v}{\partial x}\right)+\frac{\partial}{\partial y}\left(\mu \frac{\partial v}{\partial y}\right)-\frac{\partial P}{\partial y} \\
\frac{\partial(\rho u)}{\partial x}+\frac{\partial(\rho v)}{\partial y}=0
\end{array}\right.
$$

where

$P$ - pressure;

$\mu$ - dynamic viscosity;

At the entrance of the canal rate vector is horizontal and has constant value. Conditions of sticking are set on the walls of the canal. We consider flow of liquid at the exit of the canal fully developed.

Border conditions for solution of the system:

1. Conditions at the entrance of the canal: $x=0,0<y<H: u=$ const, $v=0$.

2. Conditions on the walls of the canal: $y=0, y=H, 0 \leq x \leq L: u=v=0$.

3. Conditions at the exit of the canal: $x=0,0<x<H: \frac{\partial u}{\partial x}=\frac{\partial v}{\partial x}=0$.

Control volume method is used for discretion of Navier-Stocks system in the present work. Using the method, calculated area is divided into a number of non-crossing control volumes in which hotspots can be found for calculation of designed value. Differential equation is integrated for each control volume. Power law of approximation of rate between net hotspots is used for calculation of received integrals.

SIMPLE Patankara - Spaulding algorithm is used for receiving rate areas and pressure [3].

As it can be seen from picture 1, flat canal has a complicated form that is why net chess with blocked areas in Descartes system of coordinates is used for calculation of flow area [4].

\section{Results}

Primary flow rate should be taken as $u=0.3 \mathrm{~m} / \mathrm{s}$, with physical properties of air:

$\mu=1.808 \cdot 10^{-5} \mathrm{~Pa} \cdot \mathrm{s}$ and $\rho=1.205 \mathrm{~kg} / \mathrm{m}^{3}$. Particle has the following dynamic viscosity and density: $\mu_{\mathrm{k}}=0.001005 \mathrm{~Pa} \cdot \mathrm{s}$ and $\rho_{k}=998 \mathrm{~kg} / \mathrm{m}^{3}$. Net chess is used in the calculation with dimensions $100 \times 140$ with permanent steps.

Walls of the canal are set as functional dependences so that geometric parameters $a, h, L$ were constant at different angles $\delta$.

Trajectories of particles movement in canal with diameter $d_{\mathrm{k}}=100 \mathrm{mkm}$ and $200 \mathrm{mkm}$ at primary position $y(0)=0.05 \mathrm{~m}$ and $x(0)=0 \mathrm{~m}$ are shown in picture 2 . Particles with small diameter (small mass) can smoothly flow through canal without interacting with solid border and particles with big diameters bump with solid surface and precipitate. 


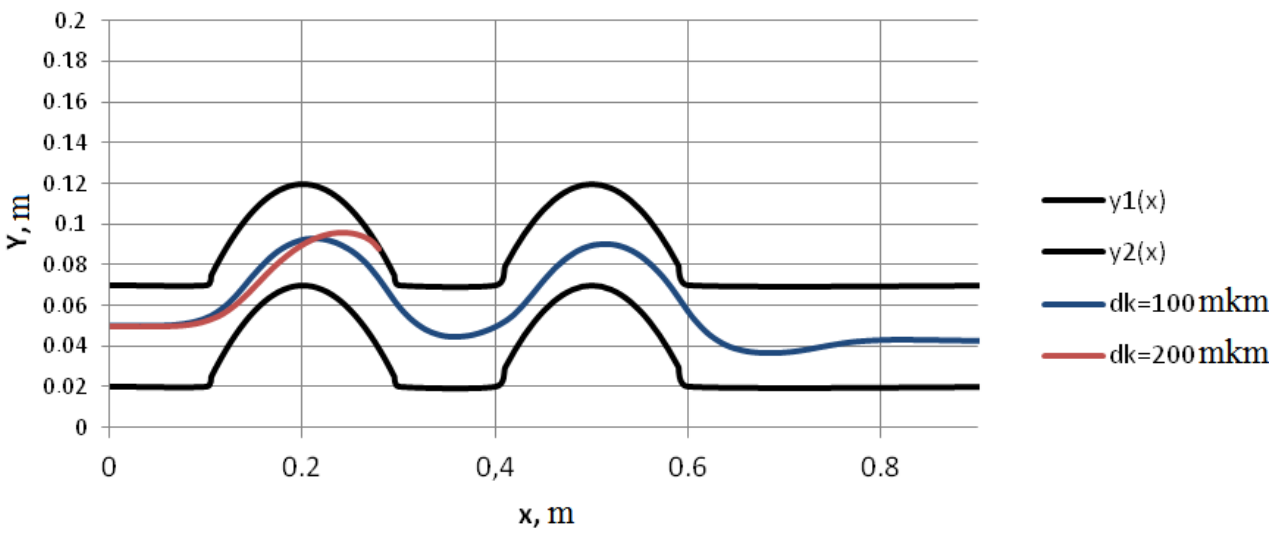

Fig. 2. Trajectory of particles movement with different masses.

In the given mathematic model influence of curvature curves of the researched canal on the particles precipitation percent of different diameter at equal border conditions of continuum flow and primary conditions for particle movement has been considered.

Share of precipitated particles in dependence of their diameter and canal curvature is shown in picture 3 (angle $\delta$ ).

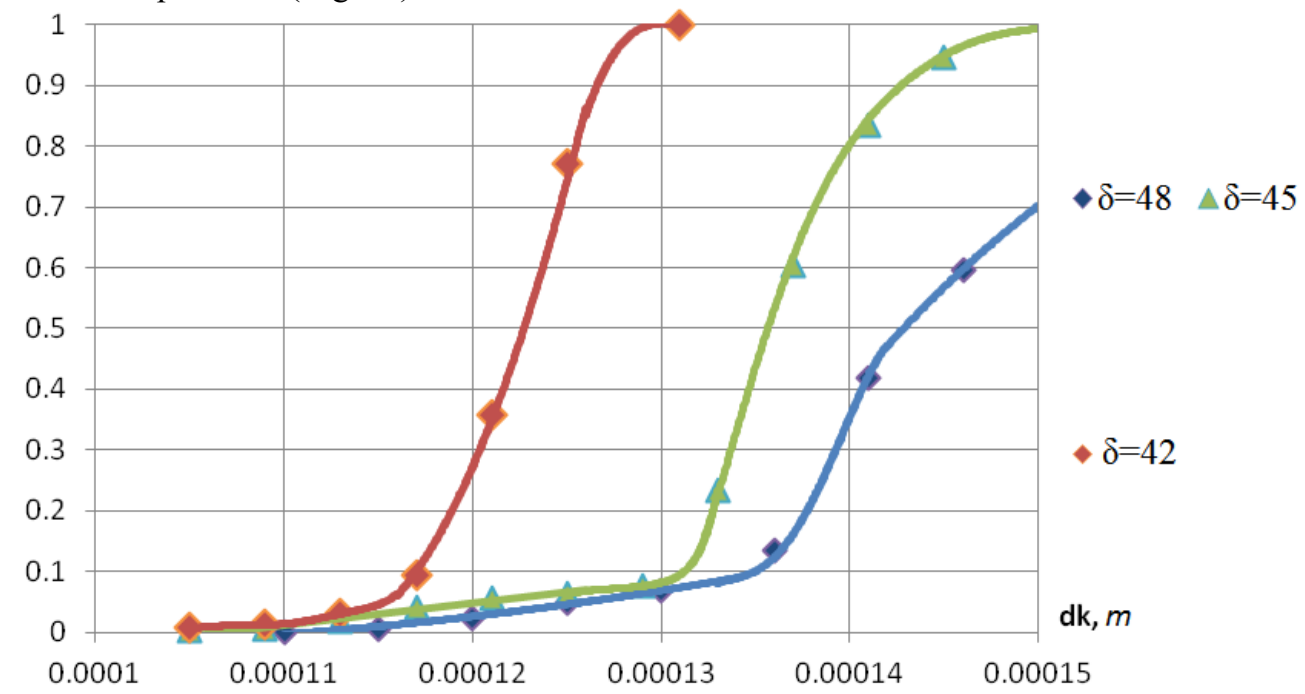

Fig. 3. Share of precipitating particles in dependence of precipitating drops on their diameter and canal curvature.

According to the received data of calculation we can conclude that increase of canal curvature leads to decrease of particles diameter where precipitation of all drops will occur. For the angle $\delta=42^{\circ}$, beginning with diameters $d_{k} \approx 130 \mathrm{mkm}$ and more, almost all the particles will precipitate on the walls of the researched canal. For the angle $\delta=45^{\circ}$ all the particles precipitate beginning with diameters $d_{k} \approx 150 \mathrm{mkm}$. 


\section{Conclusion}

Mathematic model of spherical particles movement in laminar flow of non-burnt liquid has been developed in the present work.

The result of calculations is receiving graphic dependence of precipitating drops on their diameter and canal curvature.

\section{References}

1. T. Kh. Margulova, Atomic power stations (1994)

2. M. E. Deich, Gas dynamics of two-phase media (1981)

3. S. V. Patankar, Numerical Heat Transfer and Fluid Flow (Taylor \& Francis, 1980)

4. H.G. Versteeg, W. Malalasekera, An introduction to computational fluid dynamics (Pearson Education Limited, 2007) 